\title{
WAKE EFFECTS BEHIND A FARM OF WAVE ENERGY CONVERTERS FOR IRREGULAR LONG-CRESTED AND SHORT-CRESTED WAVES
}

\author{
Peter Troch ${ }^{1}$, Charlotte Beels ${ }^{1}$, Julien De Rouck ${ }^{1}$ and Griet De Backer ${ }^{1}$
}

\begin{abstract}
The contribution of wave energy to the renewable energy supply is rising. To extract a considerable amount of wave power, Wave Energy Converters (WECs) are arranged in several rows or in a 'farm'. WECs in a farm are interacting (e.g. the presence of other WECs influence the operational behaviour of a single WEC) and the overall power absorption is affected. In this paper wake effects in the lee of a single WEC and multiple WECs of the overtopping type, where the water volume of overtopped waves is first captured in a basin above mean sea level and then drains back to the sea through hydro turbines, are studied using the time-dependent mild-slope equation model MILDwave. The wake behind a single WEC is investigated for long-crested and short-crested incident waves. The wake becomes wider for larger wave peak periods. An increasing directional spreading results in a faster wave regeneration and a shorter wake behind the WEC. The wake in the lee of multiple WECs is calculated for two different farm lay-outs, i.e. an aligned grid and a staggered grid, with varying lateral and longitudinal spacing. The wave power redistribution in and behind each farm lay-out is studied in detail using MILDwave. In general, the staggered grid results in the highest overall wave power absorption.
\end{abstract}

Keywords: wave propagation modeling; mild slope equations; wave energy converter; wake effect; farm lay-out; renewable energy

\section{INTRODUCTION}

Several wave energy converters (WECs) have been intensively studied and developed during the last decade and currently small farms of WECs are getting installed. WECs in a farm are partly absorbing, partly redistributing the incident wave power. As a result, a wake behind each WEC is created. The power absorption of each individual WEC in a farm is affected by the wakes of its neighbouring WECs.

In literature only first simplified attempts to identify the wave climate near a farm have been reported. Millar, Smith and Reeve (2006) have used the spectral wave propagation model SWAN (Booij et al. 2004) to implement an array of WECs as a single $4 \mathrm{~km}$ long partially transmitting obstacle with energy transmission ranging from $0 \%$ up till $90 \%$ of the incident wave energy. By modeling a farm as one large WEC, the redistribution of wave energy behind the individual WECs due to diffraction is not taken into account. In Venugopal and Smith (2007), WECs are modelled in a nonlinear Boussinesq wave model (MIKE 21) as individual porous structures with a prescribed porosity. In the latter study reflection and transmission characteristics are coupled through the degree of porosity of the structure, which makes the adaptation of the absorption characteristics of a WEC in a farm to the incident wave climate impossible.

In this paper the wake behind a single WEC and multiple WECs based on the overtopping principle, where waves overtop in a basin above mean sea level and where the stored water drains back to the sea through hydro turbines (Cruz 2008), is studied in a time-dependent mild-slope equation model MILDwave, developed at Ghent University (Troch 1998). In this phase-resolving model each combination of reflection and transmission characteristics, and consequently absorption characteristics, can be modelled for all individual WECs in a farm according to the methodology presented in Beels et al. (2010). This results in a more accurate representation of the wake effects in the lee of a single WEC and a farm of WECs.

In the next section the basic equations for wave generation and propagation in the mild-slope equation model MILDwave are briefly described. Furthermore the technique, developed in Beels et al. (2010), to implement a WEC of the overtopping type in MILDwave is summarized and discussed. The next section gives an overview of the wake effects behind a hypothetical WEC of the overtopping type. The last section deals with a farm of nine hypothetical WECs of the overtopping type. The overall wave power absorption is studied for two farm lay-outs, i.e. an aligned grid and a staggered grid, for varying lateral and longitudinal spacing.

${ }^{1}$ Peter.Troch@UGent.be, Dept. of Civil Engineering, Ghent University, Technologiepark 904, 9052 Ghent, Belgium 


\section{THE MILD-SLOPE WAVE PROPAGATION MODEL MILDWAVE}

\section{Wave generation and propagation}

The mild-slope equations of Radder and Dingemans (1985), which describe the transformation of linear irregular waves with a narrow frequency band over a mildly varying bathymetry (bed steepness up to 1/3 (Booij 1983)) are given in Eq. 1 and Eq. 2:

$$
\begin{gathered}
\frac{\partial \eta}{\partial t}=B \phi-\nabla \cdot(A \nabla \phi) \\
\frac{\partial \phi}{\partial t}=-g \eta
\end{gathered}
$$

where $\eta$ and $\phi$ are respectively the surface elevation and velocity potential at the free water surface, $\nabla$ is the horizontal gradient operator, $t$ is the time, $g$ is the gravitational acceleration and where

$$
\begin{gathered}
B=\frac{\bar{\omega}^{2}-\bar{k}^{2} \bar{C} C_{g}}{g} \\
A=\frac{\bar{C} C_{g}}{g}
\end{gathered}
$$

with the phase velocity $\bar{C}$ and the group velocity $\overline{C_{g}}$ for a wave with carrier angular wave number $\bar{k}$ $\left(=\frac{2 \pi}{\bar{L}}\right)$, carrier angular frequency $\bar{\omega}(=2 \pi \bar{f})$, carrier wave length $\bar{L}$ and carrier frequency $\bar{f}$. Overbar $\left(^{-}\right)$denotes that the wave characteristic is calculated for the carrier frequency.

A summary of the model features of MILDwave is presented in this section. In MILDwave waves are generated at the offshore boundary by using the source term addition method, i.e. by adding an additional surface elevation $\eta^{*}$ to the calculated value on a wave generation line for each time step (Lee and Suh 1998) given by Eq. 5:

$$
\eta^{*}=2 \eta_{i} \frac{C_{e} \Delta t}{\Delta x} \sin \theta
$$

with the water surface elevation of incident waves $\eta_{i}$, the angle of wave rays from the x-axis $\theta$ $\left(0^{\circ} \leq \theta \leq 180^{\circ}\right)$, the grid size in x-direction $\Delta x$, the time step $\Delta t$ and the energy velocity $C_{e}$.

The wave generation line is assumed to be parallel to the X-axis in Eq. 5 (Fig. 1).

It has been proven that the model of Radder and Dingemans (1985) can be used to simulate the transformation of long- and short-crested random waves (Lee and Suh 1998).

To generate long-crested irregular waves a parameterised Jonswap spectrum (Eq. 6) has been used as an input spectrum (Liu and Frigaard 1997):

$$
S(f)=\alpha H_{s}^{2} f_{p}^{4} f^{-5} \gamma^{\exp \left(-\frac{\left(f-f_{p}\right)^{2}}{2 \sigma^{2} f_{p}^{2}}\right)} \exp \left(\frac{-5}{4}\left(\frac{f_{p}}{f}\right)^{4}\right)
$$

with the significant wave height $H_{s}$, the peak wave frequency $f_{p}$, the peak enhancement factor $\gamma$, the scaling parameter $\alpha$ and the spectral width parameter $\sigma$ which equals 0.07 for $f \leq f_{p}$ and 0.09 for $f \geq f_{p}$. 


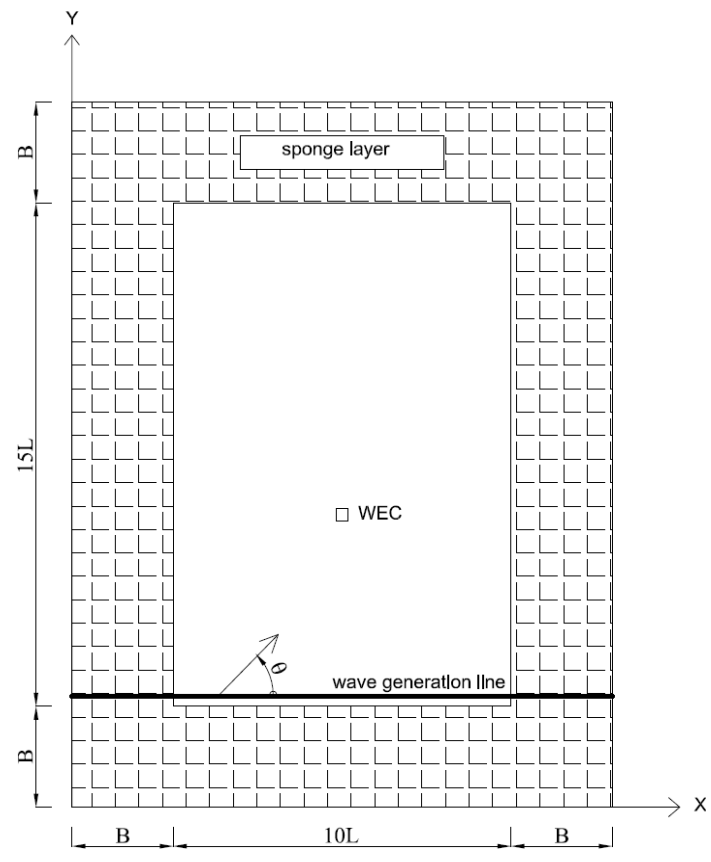

Figure 1. Definition sketch of wave basin in plan view for generation of long-crested waves.

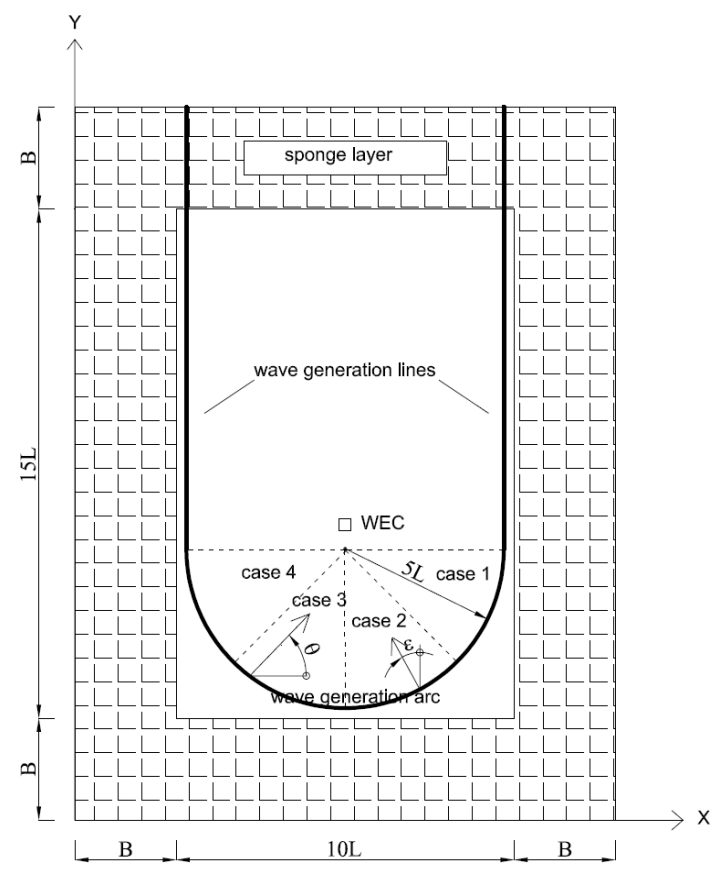

Figure 2. Definition sketch of wave basin in plan view for generation of short-crested waves.

To generate short-crested or multi-directional irregular waves, the set-up in Fig. 1 cannot be applied as not all wave components are travelling in the same direction. Lee and Yoon (2007) developed a method for internally generating multidirectional waves on an arc in a rectangular grid system using the source term addition method (Fig. 2). This technique generates wave energy much closer to the target energy than the technique of generating waves on three wave generation lines (Lee and Suh 1998), especially for a small grid size. The grid point nearest the arc is used as a wave generation point. Depending on the angle of the wave generation arc with the $\mathrm{x}$-axis, and respectively the $\mathrm{y}$-axis, the additional surface elevation is given by:

$$
\eta^{*}=\left\{\begin{array}{rr}
2 \eta_{i} \frac{C_{e} \Delta t}{\Delta y} \frac{(-\cos (\varepsilon+\theta))}{\cos \varepsilon} & \text { case } 1 \\
2 \eta_{i} \frac{C_{e} \Delta t}{\Delta x} \frac{(-\sin (\varepsilon-\theta))}{\cos \varepsilon} & \text { case } 2 \\
2 \eta_{i} \frac{C_{e} \Delta t}{\Delta x} \frac{\sin (\varepsilon+\theta)}{\cos \varepsilon} & \text { case } 3 \\
2 \eta_{i} \frac{C_{e} \Delta t}{\Delta y} \frac{\cos (\varepsilon-\theta)}{\cos \varepsilon} & \text { case } 4
\end{array}\right.
$$

with $\varepsilon$ the angle between the line normal to the wave generation curve and the $\mathrm{x}$-axis for case 1 and 4 , and respectively the y-axis for case 2 and 3. $\varepsilon$ is indicated on Fig. 2 for case 2.

On the left, and respectively the right generation line, the additional surface elevation is given by Eq. 8 and Eq. 9:

$$
\begin{gathered}
\eta^{*}=2 \eta_{i} \frac{C_{e} \Delta t}{\Delta x} \cos \theta \\
\eta^{*}=2 \eta_{i} \frac{C_{e} \Delta t}{\Delta x}(-\cos \theta)
\end{gathered}
$$

When the wave direction is not pointing to the inner domain on a part of the wave generation arc or on one of the wave generation lines, no waves are generated in those cells. For example, when $\theta=45^{\circ}$ no waves are generated on the right generation line and on the first part of the arc (case 1).

Equation 10 gives the directional spreading function $D(f, \theta)$ to generate short-crested waves (Mitsuyasu et al. 1975): 


$$
D(f, \theta)=\frac{2^{2 s-1}}{\pi} \frac{\Gamma^{2}(s+1)}{\Gamma(2 s+1)} \cos ^{2 s}\left(\frac{\theta-\theta_{o}}{2}\right)
$$

with the mean wave angle $\theta_{o}$. The directional spreading parameter $s$ gives the degree of directional energy concentration. For wind seas (like the North Sea), the spectrum is rather broad and the value of $s$ is rather low. The parameter $s$ can be related to the frequency by Eq. 11 (Goda and Suzuki 1975):

$$
s= \begin{cases}\left(f / f_{p}\right)^{5} s_{\max } & : \quad f \leq f_{p} \\ \left(f / f_{p}\right)^{-2.5} s_{\max } & : \quad f \geq f_{p}\end{cases}
$$

The parameter $s_{\max }$ is the peak value of the spreading parameter. Fixed values for $s_{\max }$ are given in Eq. 12 for wind and swell waves (Goda and Suzuki 1975):

$$
s_{\max }=\left\{\begin{array}{lll}
10 & : & \text { wind waves } \\
25 & : & \text { swell } \text { with short decay distance } \\
75 & : & \text { swell with long decay distance }
\end{array}\right.
$$

The effect of the fully reflective domain boundaries is negligible because absorbing sponge layers (Larsen and Dancy 1983) at the outside boundaries (Fig. 1 and Fig. 2) are significantly dissipating the incoming wave energy by multiplying the calculated surface elevations on each new time step with an absorption function $S(b)$ (reflection coefficient smaller than $5 \%$ for $\mu=1.04$ and $a_{n}=60$ in Eq. 13):

$$
S(b)=\frac{1}{\exp \left(\left(\mu^{-b}-\mu^{-B}\right) \ln a_{n}\right)}
$$

with the length of the sponge layer $B$ and the distance from the outside boundary $b$, both expressed in number of cells. In general, a length of $2.5 L_{\max }$ (Lee and Suh 1998), where $L_{\max }$ is the maximum wave length resulting from the cut-off incident frequency spectrum, is sufficient.

A finite difference scheme, as described in Brorsen and Helm-Petersen (1998), is used to solve Eq. 1 and Eq. 2. The domain is divided in grid cells (uniform grid) with dimensions $\Delta x$ and $\Delta y$ and central differences are used for spatial derivates. The grid spacing $\Delta x=\Delta y$ is chosen so that $\frac{L_{\text {min }}}{20} \leq \Delta x=\Delta y \leq \frac{L_{\text {min }}}{10}$ ( $L_{\text {min }}=$ shortest wave length (maximum frequency) for irregular waves) and the time step meets the Courant-Friedrichs-Lewy criterion, $\Delta t \leq \frac{\Delta x}{C}$, to guarantee a stable and consistent result.

Wave generation starts from quiescent water conditions at $t=0$. A slow start of wave generation is obtained by multiplying the wave generation by $\tanh \left(\frac{0.5 t}{T}\right)$ (Suh et al. 2001), with the wave period $T$. Each time step, $\eta$ and $\phi$ are calculated in the centre of each grid cell. Consequently, waves are gradually propagating through the domain.

\section{Sponge layer technique}

A WEC is a fixed or moored (rigidly or slack) structure that is able to extract wave power by its specific design. The amount of produced power by the WEC, as a function of the incident wave climate, is generally determined through a numerical or physical model of the WEC and summarized in a power matrix. In this section a technique will be described to simulate WECs based on the overtopping principle which capture the water volume of overtopped waves in a basin above sea level and consequently absorb a part of the incident wave power comparable to a porous structure. Incident waves are partly reflected on the WEC, are partly overtopped in the basin and consequently absorbed and partly transmitted under and around the structure. Therefore, a WEC is only absorbing a certain amount of the incident wave power available over the width of the device. The degree of absorption, as a function of the incident wave climate, can be derived based on a power matrix from the WEC developer. Also, the amount of reflection from the structure can be specified by the developer. In most 
cases the reflection will be rather small as a WEC is designed to absorb wave power and to reduce energy loss by reflection and transmission.

A WEC is implemented in MILDwave as an array of cells (covering the spatial extensions of the WEC) that have been assigned a given degree of absorption using the sponge layer technique. Absorption function $S(x)$ or $S(y)$ define the absorption coefficient $S$ attached to each cell of the WEC in the $\mathrm{x}$-direction, and the y-direction, respectively (Fig. 1). By changing the values of the absorption coefficients or the number of absorbing cells, the degree of reflection and transmission and consequently absorption of the porous structure can be changed. When all cells have a constant absorption coefficient $S(x)=S(y)=0$, respectively $S(x)=S(y)=1$, all incident wave power is reflected, respectively transmitted. A structure with a value of $S$ equal to 0 represents a fully reflective structure. Cells with $S$ equal to 1 are water cells. When the absorption coefficient is varying between 0 and 1 , the incident waves are partly reflected, absorbed and transmitted. When assuming a constant absorption coefficient $S$ for all cells of the WEC, the amount of reflection, transmission and absorption are coupled, as seen in Venugopal and Smith (2007). To avoid this coupling, the shape of the absorption function $S(y)$ through the WEC (when the direction of wave propagation = y-direction) is changed (Beels et al. 2010, 2010b). This way the degree of absorption (and consequently transmission) of the WEC, given in the power matrix of the WEC, can be tuned for a fixed amount of reflection on the WEC as specified by the developer.

To tune the reflection, transmission and consequently absorption characteristics of a WEC, a structure composed of a series of absorbing cells has been implemented in a numerical test flume (Fig. 3). The structure has a length equal to the length of the WEC and a width equal to the wave flume width.

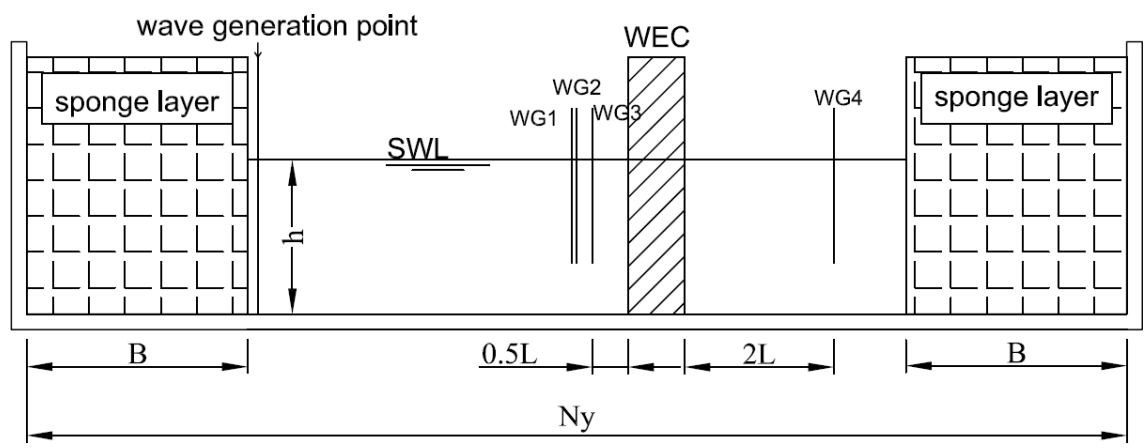

Figure 3. Definition sketch of numerical test flume - cross section.

To determine the amount of reflection on and transmission through the absorbing structure, four wave gauges are placed in the wave flume (Fig. 3). Three wave gauges are installed half a wave length in front of the structure to measure the incident significant wave height $H_{s, i}$ and the reflected significant wave height $H_{s, r}$. The distances between the wave gauges are derived using Mansard and Funke (1980) in order to perform a reflection analysis. One wave gauge is placed two wave lengths behind the WEC to measure the transmitted significant wave height $H_{s, t}$. The degree of absorbed power $\frac{P_{a}}{P_{i}}$ in deep water is calculated using (conservation of energy):

$$
\frac{P_{a}}{P_{i}}=1-K_{r}^{2}-K_{t}^{2}
$$

with the absorbed wave power $P_{a}$, the incident wave power $P_{i}$, the reflection coefficient $K_{r}$ $=H_{s, r} / H_{s, i}$ and the transmission coefficient $K_{t}=H_{s, t} / H_{s, i}$.

In this paper a hypothetical WEC of the overtopping type of $36 \mathrm{~m} \times 36 \mathrm{~m}$ has been used as an example. The hypothetical WEC has a prescribed capture ratio of $45 \%$ and a pre-specified overall 
reflection coefficient of 0.14 for a sea state with $H_{s}=1 \mathrm{~m}$ and $T_{p}=5.2 \mathrm{~s}$. The capture ratio is defined as the ratio between the absorbed power and the wave power incident on a wave-front width equal to the width of the WEC. The amounts of reflection and transmission have been tuned in a wave flume with a width equal to $36 \mathrm{~m}$.

The time series at the wave gauges are sampled with a frequency of $20 \mathrm{~Hz}$. In all tests, a uniform deep water depth $h$ is used to avoid energy dissipation due to bottom friction and wave breaking. Waves with a peak period of $5.2 \mathrm{~s}$ and a significant wave height of $1 \mathrm{~m}$ have the highest frequency of occurrence in the southern part of the North Sea and are considered in this study as the basic case.

\section{WAKE EFFECTS}

\section{A single WEC}

The wake behind the specified WEC has been studied for four different sea states with $H_{s}=1 \mathrm{~m}$ (Table 1). The values of the absorption coefficient through the WEC have been tuned separately for each peak wave period to obtain a capture ratio of $45 \%$, since the absorption is frequency dependent (Beels et al. 2010). Depending on the type of WEC, the capture ratio may vary with mean incident wave direction. Only head-on waves are considered in this work. Irregular long-crested and short-crested waves are generated during respectively $3500 \mathrm{~s}$ and $10000 \mathrm{~s}$ with a time step of $0.05 \mathrm{~s}$.

\begin{tabular}{|l|c|c|}
\hline \multicolumn{3}{|c|}{ Table 1. Sea states. } \\
\hline Test & $T_{p}[s]$ & $s_{\max }[-]$ \\
\hline A & 5.2 & - \\
B & 7.8 & - \\
C & 5.2 & 75 \\
D & 5.2 & 10 \\
\hline
\end{tabular}

The disturbance coefficients $K_{d}=H_{s, d} / H_{s, i}$, where $H_{s, d}$ is the numerically calculated disturbed significant wave height and $H_{s, i}$ is the numerically calculated incident significant wave height at the wave generating boundary, in a wave basin with a single WEC (with test set-up as shown in Fig. 1) for long-crested waves (head-on) with a peak wave period of respectively $5.2 \mathrm{~s}$ and $7.8 \mathrm{~s}$ (test cases A and B) are shown in Fig. 4(a) and Fig. 4(b). On these figures only the useful domain without sponge layers is shown. The length and the width of the useful domain are equal to respectively $15 \mathrm{~L}$ and $10 \mathrm{~L}$ where $L=100 \mathrm{~m}$ (deep water wave length for $T_{p}=7.8 \mathrm{~s}$ ) for all test cases. The position of the WEC is indicated by a white square.

A small wave height increase at the edges of the wake due to diffraction is observed for both wave periods. The wave height decrease behind the WEC is smaller for a peak wave period of $7.8 \mathrm{~s}$, approximately $0.175 \mathrm{~m}$, compared to $0.225 \mathrm{~m}$ for a peak wave period of $5.2 \mathrm{~s}$. Furthermore, the shadow zone is wider for a higher wave period. This result indicates that the peak wave length will influence the optimal pattern of a farm where a WEC should avoid the centre of the wake location of a neighbouring WEC. If swell waves are dominating, the peak wave period with the highest frequency of occurrence should be taken into account when designing the optimal pattern of a farm.

On the other hand, if wind waves are dominating, the shadow zone will be smaller and less visibly dependent on the directional spreading of the short-crested waves. The disturbance coefficients around a single WEC are calculated for short-crested waves with a mean wave direction of $90^{\circ}$ (Fig. 4(c) and Fig. 4(d)) where the directional spreading is increasing from approximately $9^{\circ}\left(s_{\max }=75\right)$ up to $24^{\circ}$ $\left(s_{\max }=10\right)$ for the peak period (as we assume a frequency dependent spreading parameter (Eq. 11)). To generate short-crested waves a set-up as shown in Fig. 2 has been used which results in a useful domain indicated with black lines on Fig. 4(c) and Fig. 4(d).

In the case of short-crested waves a wake is still observed, as the waves need to travel some distance before the effects of regeneration, caused by the directional spreading of the waves and wave diffraction around the WEC, are apparent. In Fig. 4(c) a long shadow zone occurs behind the WEC, comparable with the one observed in Fig. 4(a), as the directional spreading is still quite small. In Fig. 4(d) the shadow zone is shorter due to a faster regeneration behind the WEC from waves with wave angle between approximately $90^{\circ} \mp 24^{\circ}$. 


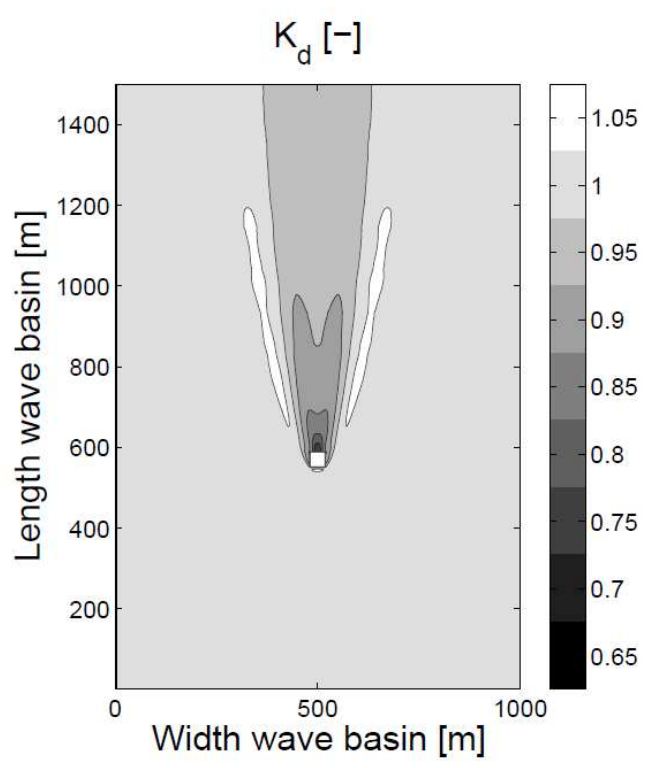

(a) Long-crested waves with $T_{p}=5.2 \mathrm{~s}$.

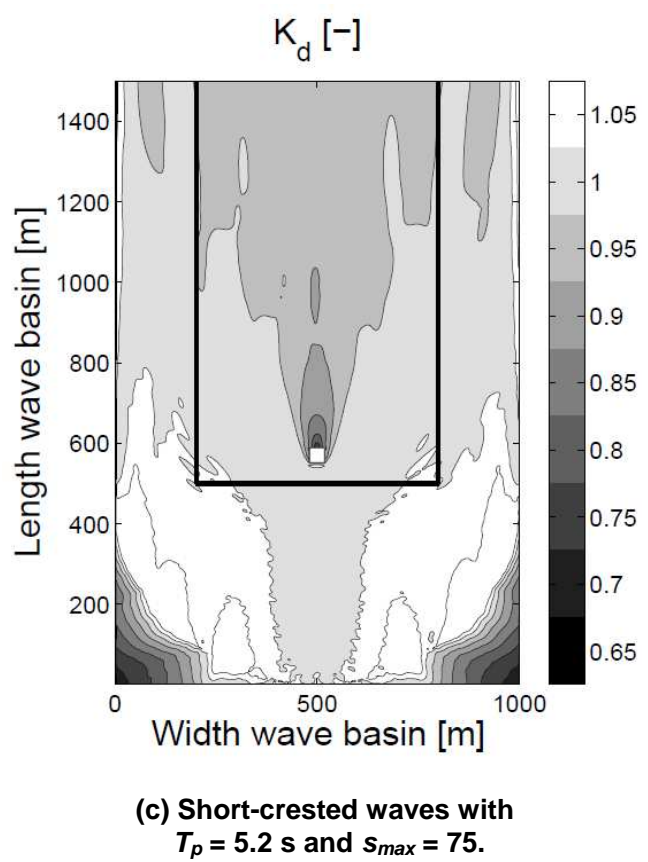

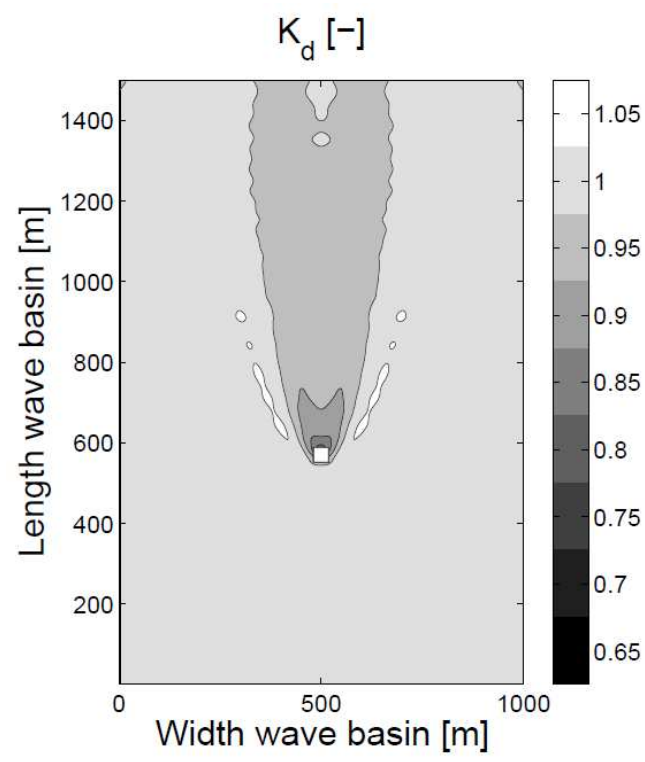

(b) Long-crested waves with $T_{p}=7.8 \mathrm{~s}$.

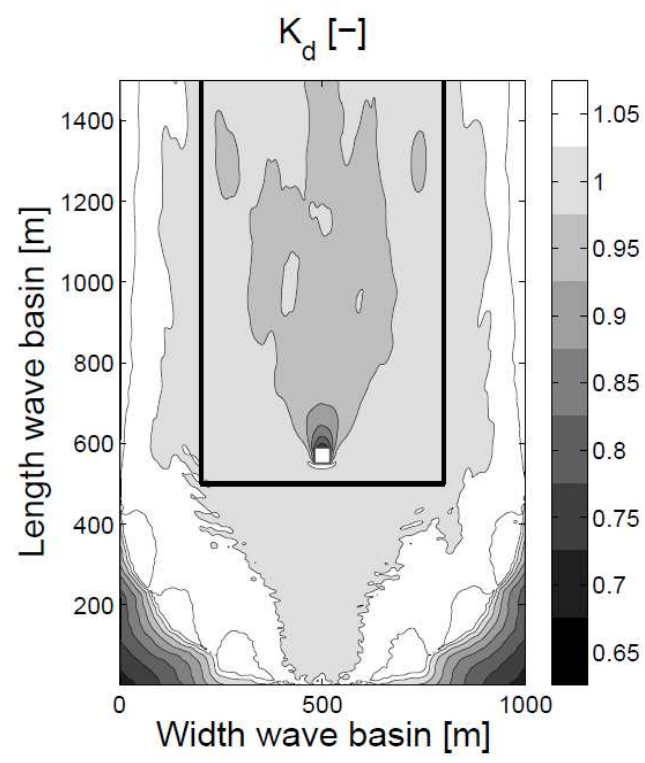

(d) Short-crested waves with $T_{p}=5.2 \mathrm{~s}$ and $s_{\max }=10$.

Figure 4. Calculated disturbance coefficient $K_{d}$ in a wave basin with a single WEC (capture ratio $=45 \%$ ) for irregular long-crested waves (head-on) with respectively $T_{p}=5.2 \mathrm{~s}(\mathrm{a}), T_{p}=7.8 \mathrm{~s}(\mathrm{~b})$ and for irregular shortcrested waves (head-on - useful domain indicated with black lines) with $T_{p}=5.2 \mathrm{~s}$ and respectively $s_{\max }=75$ (c), $s_{\max }=10$ (d).

It is clear that regeneration behind a device depends on the peak period and directional spreading of the incident wave climate. The higher the peak period and the higher the directional spreading, the faster waves are regenerated behind the WEC. Regeneration by wind and energy dissipation by wave breaking are not taken into account in this paper. The smaller the directional spreading the longer the shadow zone becomes. Therefore in the next paragraph, when studying the power absorption of a farm, only swell waves have been considered as those waves cause the largest wake behind the WEC and consequently the largest impact on the neighbouring WECs in the farm. As a consequence, the calculated amount of absorbed power will be conservative. 


\section{A farm of nine WECs}

The understanding of the impact of one WEC on the wave climate is very crucial in determining the capture ratio of the neighbouring WECs in a farm. Since a single WEC is reducing the incident wave height in its lee, the capture ratio of a WEC installed in its lee will decrease or increase depending on the magnitude of the wave height reduction. The latter reduction is determined by the dimensions and capture ratios of the surrounding WECs and the in-between distance of the WECs in the farm. The dimensions of a WEC define the diffracted wave pattern around the WEC, while the magnitude of wave height reduction in the diffracted wave pattern is depending on its capture ratio. The higher the distance between the WECs the more energy is travelling inside the farm. Furthermore, the distance between the WECs in a row and the in-between distance of the rows will determine the total impact of the farm on the wave climate and on the surrounding users of the sea. The distance between the WECs in a farm should be as small as possible to reduce the cost of the farm. Furthermore, a minimal distance equal to twice the WEC dimension is needed to remove a damaged WEC from the farm.

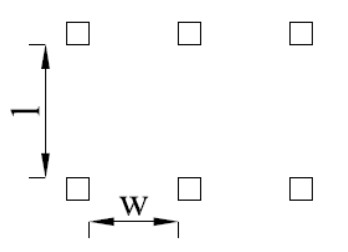

Aligned grid

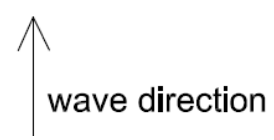

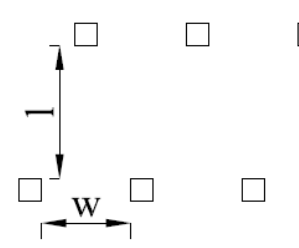

Staggered grid

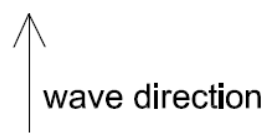

Figure 5. Aligned and staggered grid lay-out.

In this section the influence of the lateral and longitudinal spacing, respectively $w$ and $l$, on the amount of absorbed wave power is studied for incident irregular long-crested waves with $H_{s}=1 \mathrm{~m}$ and $T_{p}=5.2 \mathrm{~s}$ (test case A). Two different lay-outs (Fig. 5) with nine hypothetical WECs, as defined in the previous sections, have been compared; a lay-out with 3 identical rows (an aligned grid), where the WECs are placed right behind each other and a lay-out where the first and third row are identical, but where the second row is shifted (a staggered grid). The length and the width of the wave basin defined in Fig. 1 have been extended to obtain a sufficiently large domain to generate long-crested head-on waves with a peak wave period of $5.2 \mathrm{~s}$. A hypothetical power matrix for irregular long-crested incident waves with $T_{p}=5.2 \mathrm{~s}$ as shown in Fig. 6 has been used as an example. The power matrix is showing the ratio between the absorbed wave power and the incident wave power and not between the electrical power output and the incident wave power. This means that losses in turbines, generator, ... are ignored. The capture ratio has been plotted for significant wave heights between $0 \mathrm{~m}$ and $1 \mathrm{~m}$. No variation with wave period has been considered as only irregular long-crested waves with $H_{s}=1 \mathrm{~m}$ and $T_{p}=5.2 \mathrm{~s}$ have been generated. Each capture ratio (Fig. 6) has been tuned in a wave flume as explained before. The overall reflection coefficient $K_{r}$ was assumed smaller than 0.15 in all cases. In that case the resulting amount of reflected wave power was smaller than $2 \%$ of the incident wave power. Due to the technique of uncoupling reflection and transmission, it was possible to keep the reflection small for each defined capture ratio and tune the amount of transmission and consequently the amount of absorption. The latter uncoupling was needed to implement a procedure of adaptive absorption (adapting the capture ratio of each WEC in the farm to its incident wave power without changing the amount of reflection) to simulate a farm. 


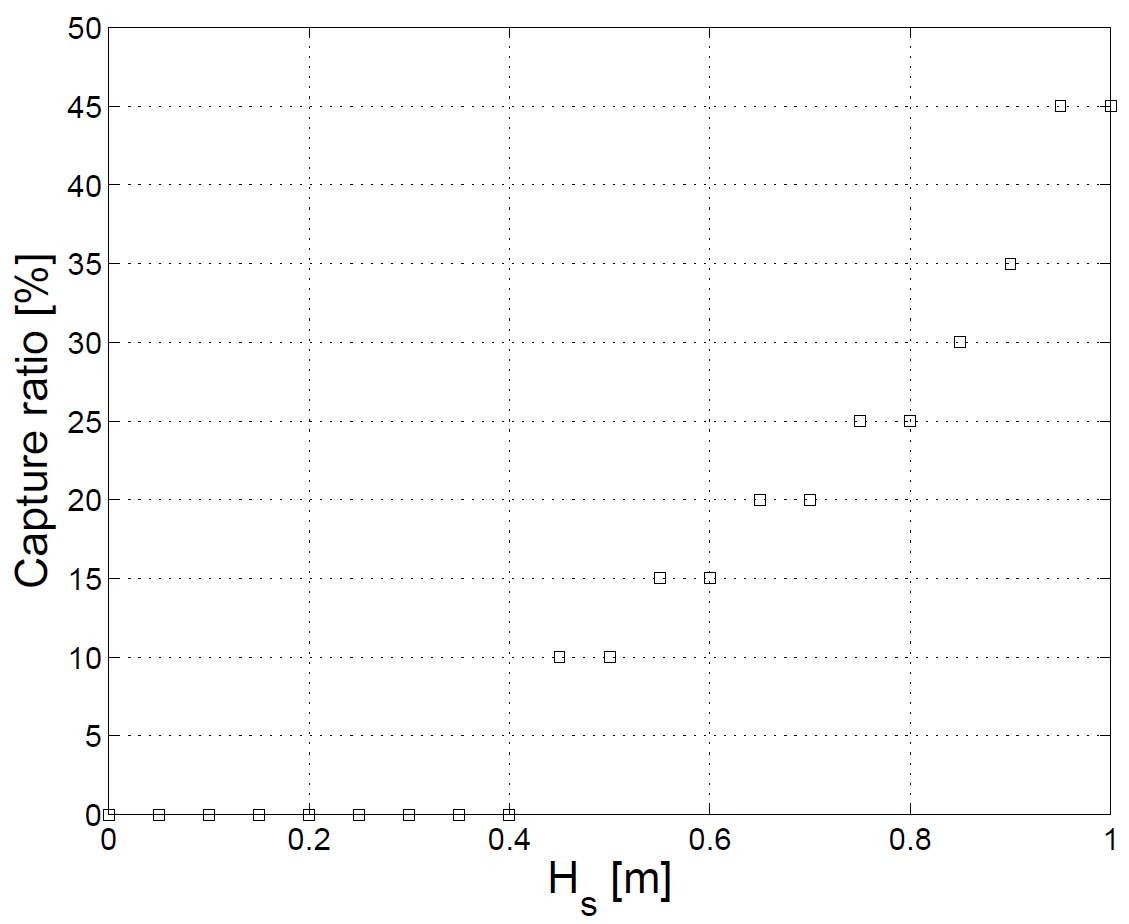

Figure 6. Capture ratio as a function of significant wave height $H_{s}$.

In a first step, only three WECs, with an in-between distance $w$ of respectively $2 \mathrm{D}, 4 \mathrm{D}$ and $6 \mathrm{D}$ where D is the width of the WEC, in the first row with the same capture ratio as an isolated WEC (45 $\%$ ), have been installed. The capture ratio of the WECs that will be installed in the second row, with $l$ respectively $2 \mathrm{D}, 4 \mathrm{D}, 6 \mathrm{D}$ and $20 \mathrm{D}$, is derived by measuring the wave height on the positions of the WECs and by using Fig. 6. In a second step, WECs in the two first rows are installed in the wave basin and the wave height is measured on the positions of the WECs in the third array. The average wave height on the WEC positions has been used to define its capture ratio. 


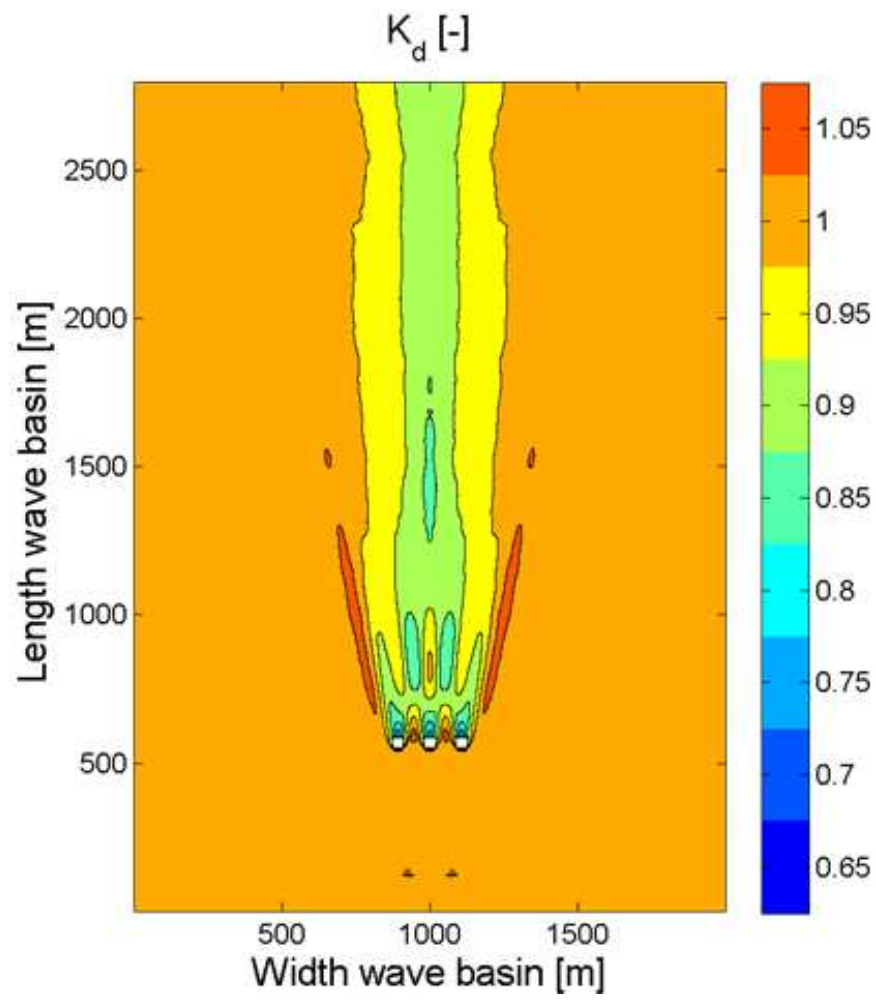

(a) $\mathbf{w}=2 \mathrm{D}$.

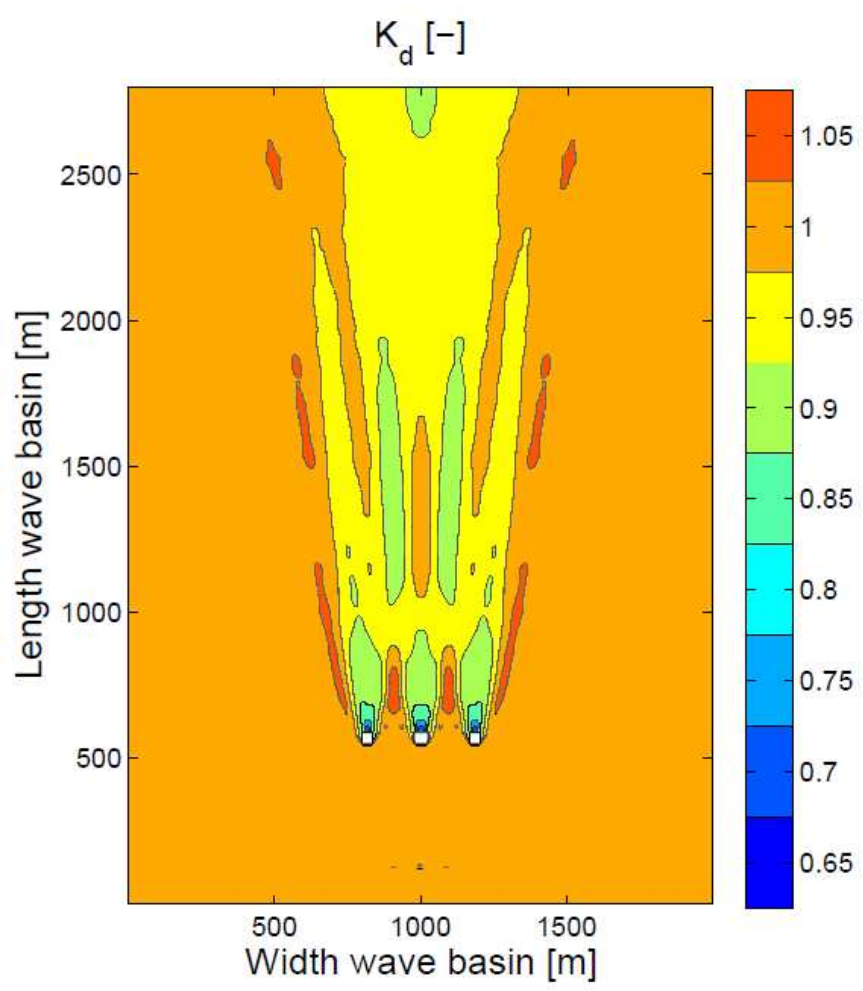

Figure 7 - for caption, see next page

(b) $w=4 D$. 


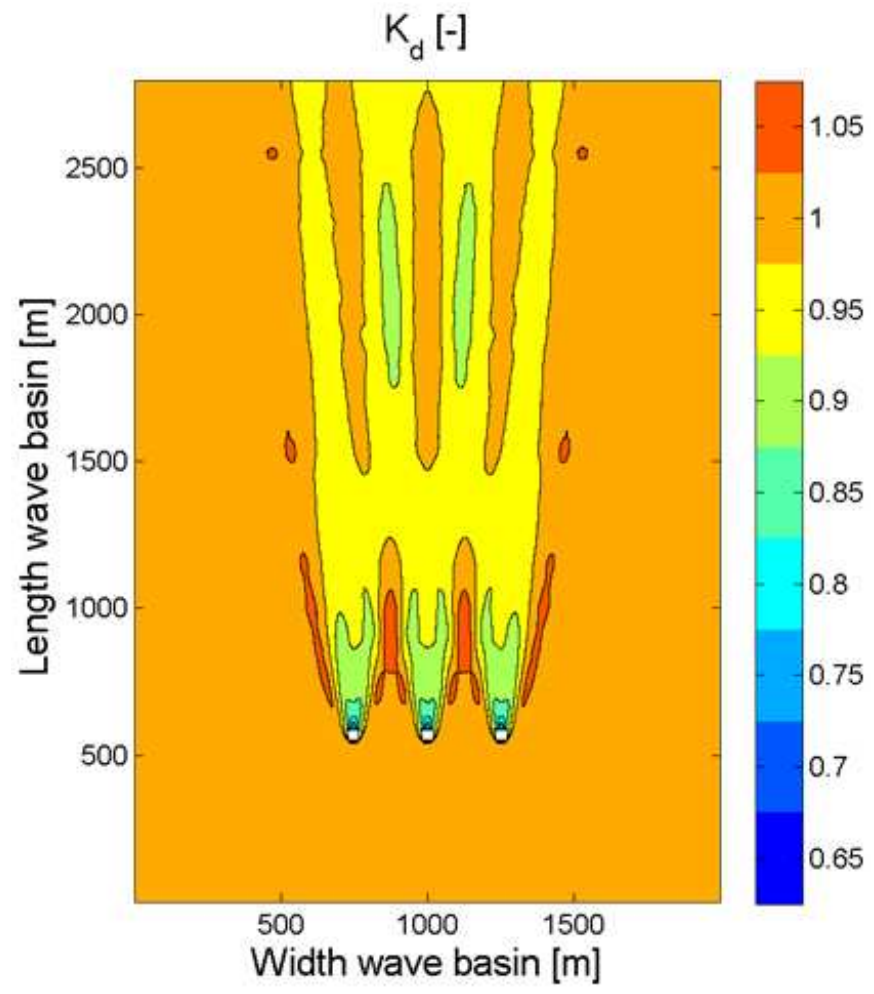

(c) $w=6 D$.

Figure 7. Calculated disturbance coefficient $K_{d}$ in a wave basin with 3 hypothetical WECs with an in-between distance of respectively (a) 2D, (b) $4 D$, (c) $6 D$ with $D=36 \mathrm{~m}$ for irregular long-crested waves (head-on) with $T_{p}=5.2 \mathrm{~s}$.

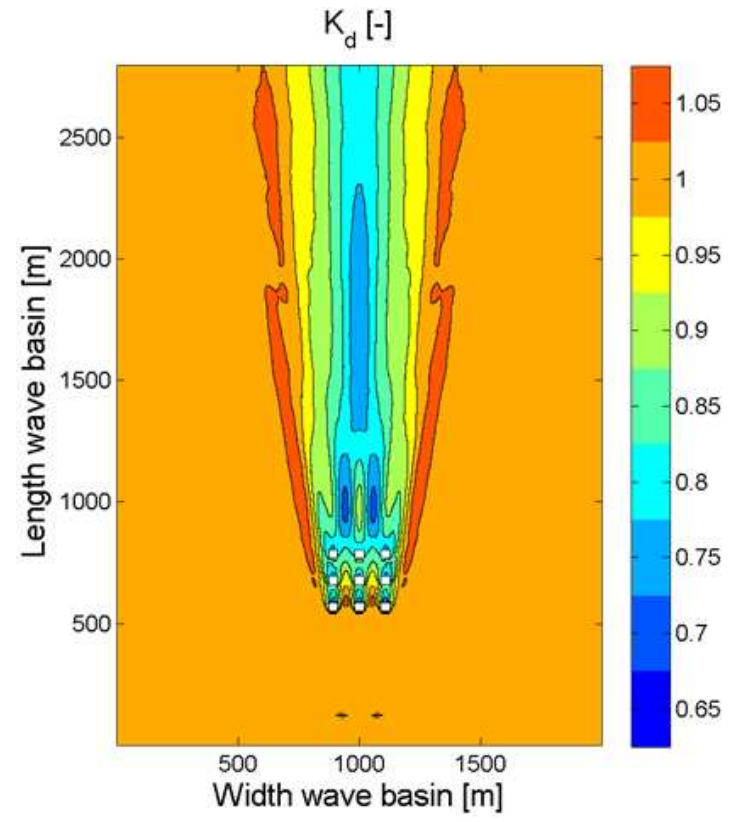

(a) Aligned grid.

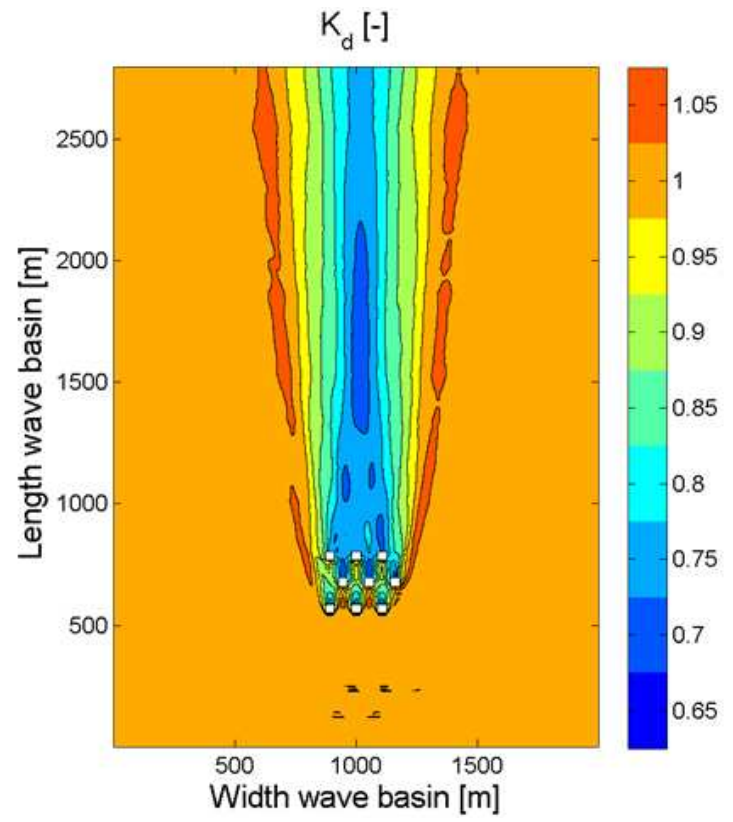

(b) Staggered grid.

Figure 8. Calculated disturbance coefficient $K_{d}$ in a wave basin with 9 hypothetical WECs with an inbetween distance of 2D with $\mathrm{D}=36 \mathrm{~m}$ for irregular long-crested waves (head-on) with $T_{p}=5.2 \mathrm{~s}$. 
The dimensionless calculated disturbed significant wave heights $K_{d}$ in a wave basin with only the first row of WECs installed, is given in Fig. 7 for test case A. Again, only the useful domain without sponge layers is shown and the positions of the WECs are indicated by white squares. The higher the inbetween distance the more energy is travelling between the WECs. The closer the WECs in the first row are installed, the faster the interaction between the individual wakes in the lee of that row.

The dimensionless calculated disturbed significant wave heights $K_{d}$ for both lay-outs (aligned grid and staggered grid) are given in Fig. 8 for test case A and a lateral and longitudinal spacing of 2D. For both lay-outs waves are simulated during $3500 \mathrm{~s}$ (time step $0.05 \mathrm{~s}$ ) in a domain of 3400 cells (width) $\mathrm{x}$ 3500 cells (length) which resulted in a computational time of 24 hours (Intel Core 2 CPU @ $2.40 \mathrm{GHz}$ 3 GB RAM).

The wave height in front of the second and third row is much higher for the staggered grid, which resulted in a higher capture ratio for the WECs in those rows (capture ratio of respectively $45 \%$ and 35 $\%$ for the second and third row) compared to the aligned grid (capture ratio of $30 \%$ for the second and third row).

Both lay-outs are compared in more detail in Fig. 9 and Fig. 10 with a lateral cross section right behind the farm on $\mathrm{y}=805 \mathrm{~m}$, and respectively a longitudinal cross section behind the farm on $\mathrm{x}=$ $1000 \mathrm{~m}$ shown for both lay-outs.

The wave height is lower (higher wave height reduction) behind the staggered grid compared to the aligned grid due to the higher capture ratio of the WECs in the second and third row. When a constant capture ratio (no adaptive absorption) would be assumed for all WECs in the farm, the difference between both lay-outs would be very small.

In Fig. 9 the cross section is symmetric for the aligned grid, as the lay-out is symmetric as well. The wake behind both lay-outs is gradually filled up behind the farm (Fig. 10).

The amount of absorbed power of an aligned and a staggered grid with varying in-between distances is calculated in the next sections for irregular long-crested waves with $H_{s}=1 \mathrm{~m}$ and $T_{p}=5.2$ s. The results are conservative as the considered incident waves are rather small and no directional spreading of the incident waves has been considered.

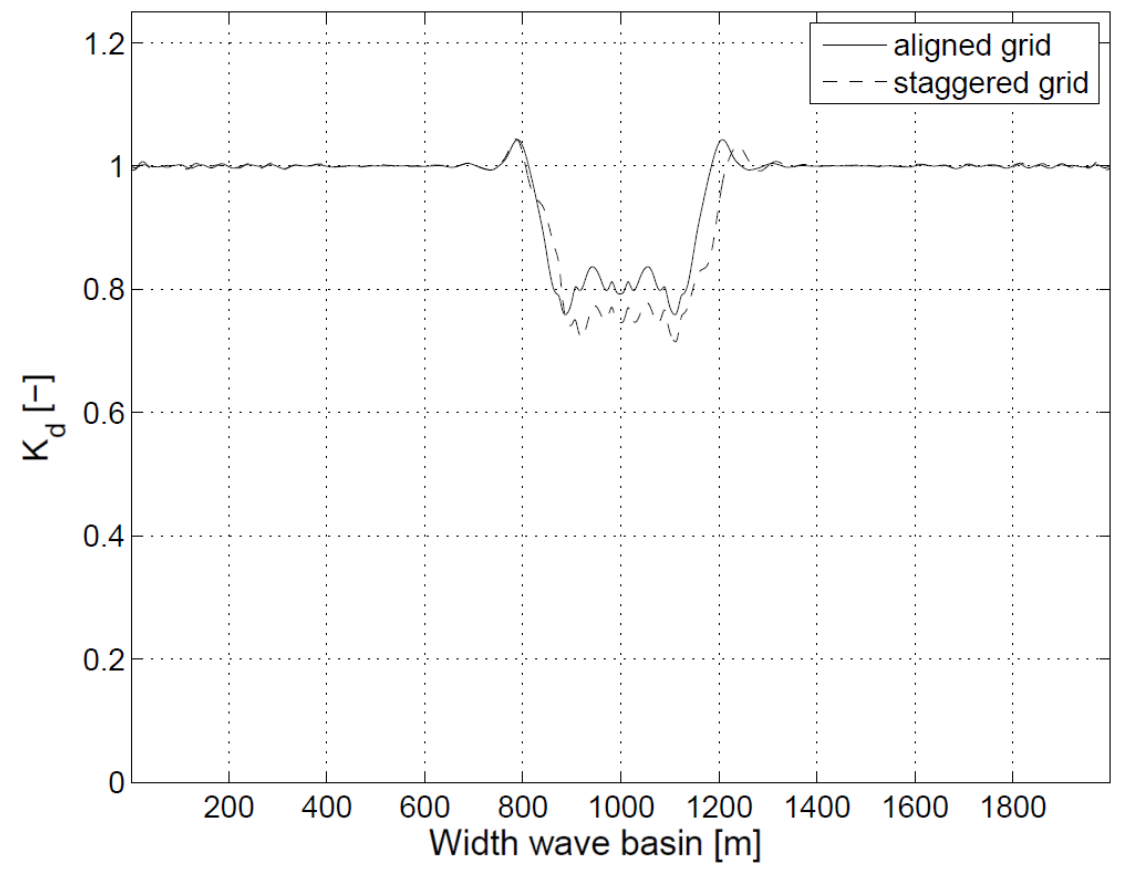

Figure 9. Calculated disturbance coefficient $K_{d}$ in a lateral cross section at y= $805 \mathrm{~m}$ for an aligned and a staggered grid with a lateral and longitudinal spacing of 2D (Fig. 8). 


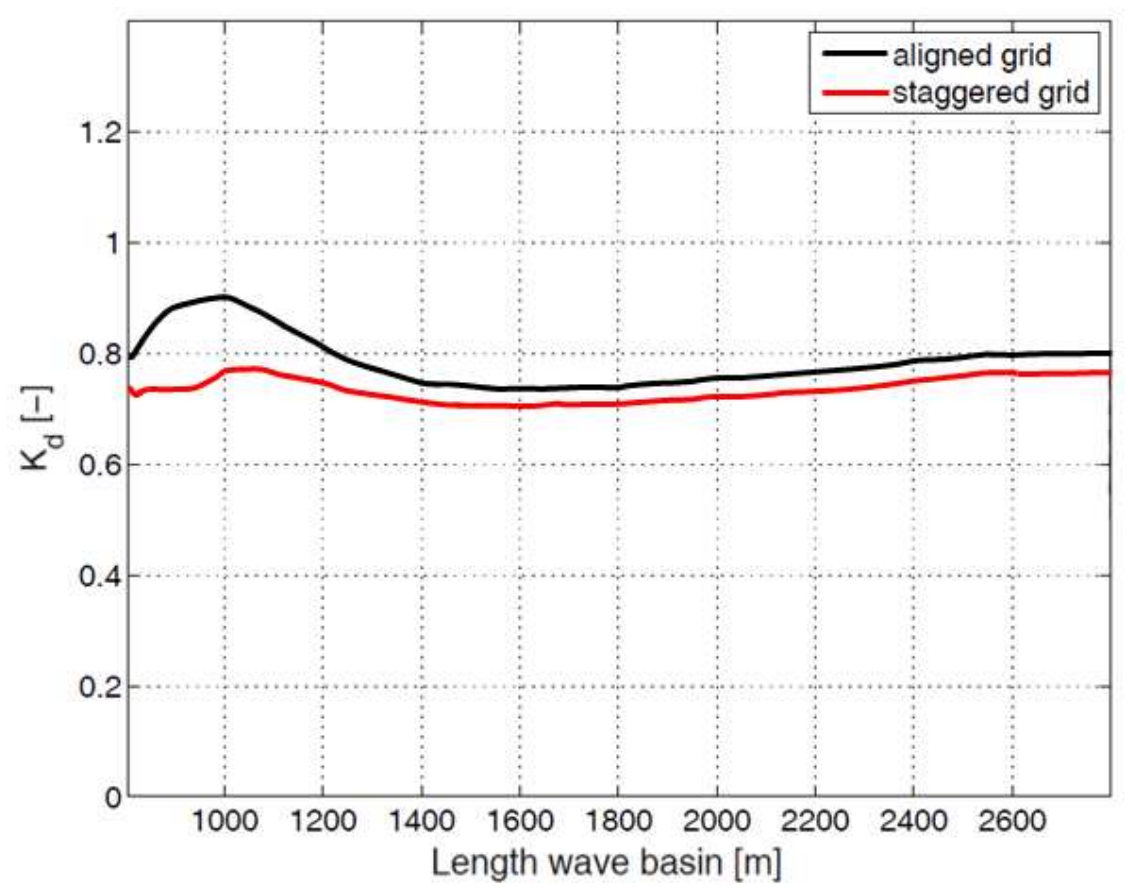

Figure 10. Calculated disturbance coefficient $K_{d}$ in a longitudinal cross section at $\mathrm{x}=1000 \mathrm{~m}$ behind an aligned grid and a staggered grid with a lateral and longitudinal spacing of 2D (Fig. 8).

\section{Aligned grid}

Table 2(a) shows the loss of the amount of absorbed power of the farm compared to a theoretical farm of nine independent WECs, for the considered in-between distances. The theoretical farm is absorbing 9 times $P_{s}$, the power absorbed by a single WEC (capture ratio of $45 \%$ ). The loss of the amount of absorbed power is expressed in a multiple of $P_{s}$. It is expected that the power loss decreases with increasing in-between distance. However, interaction between the wakes results in some deviations. The small wave height increase at the edges of the wake, observed in Fig. 4(a) and 4(b), can cause a higher wave height behind a row of WECs compared to the incident wave height (positive interaction of the wakes).

When $w=4 \mathrm{D}$ or $6 \mathrm{D}$, the power loss is comparable. For a lateral spacing $w=2 \mathrm{D}$ and a longitudinal spacing $l=4 \mathrm{D}$ or $6 \mathrm{D}$, positive interaction of the individual wakes occurs. Consequently, a lower loss of power occurs compared to an aligned grid with $w=2 \mathrm{D}$ and $l=20 \mathrm{D}$. The highest power loss $\left(3.11 P_{s}\right)$ is seen for $w=4 \mathrm{D}$ or $6 \mathrm{D}$, and $l=2 \mathrm{D}$. In those cases the WECs in the second and third row have a capture ratio of $30 \%$. When $w=6 \mathrm{D}$ and $l=20 \mathrm{D}$ only $0.70 P_{s}$ of power is lost. In that case all WECs in the farm have a capture ratio of $45 \%$.

Not only the total absorbed power of the farm is important. The absorbed power per $\mathrm{km}^{2}$ should be studied as well, as there may be space limitations for installing a farm of WECs. The smaller the inbetween distances the higher the absorbed power per $\mathrm{km}^{2}$ (Table 3(a)).

As the difference between loss of power for a lateral spacing of $4 \mathrm{D}$ and $6 \mathrm{D}$ is rather small in comparison to $2 \mathrm{D}$, a lateral spacing of $2 \mathrm{D}$ is not preferable. A lateral spacing of $4 \mathrm{D}$ is preferred in comparison to $6 \mathrm{D}$ as the power per $\mathrm{km}^{2}$ is higher. 


\begin{tabular}{|c|c|c|c|}
\hline (a) & \multicolumn{3}{|c|}{ Aligned grid. } \\
\hline$I / w$ & $2 \mathrm{D}$ & $4 \mathrm{D}$ & 6D \\
\hline $2 \mathrm{D}$ & 3.02 & 3.11 & 3.11 \\
\hline $2 \mathrm{D}$ & 2.14 & 2.64 & 2.64 \\
\hline 6D & 1.70 & 2.11 & 2.18 \\
\hline $20 D$ & 2.58 & 0.92 & 0.70 \\
\hline (b) & \multicolumn{3}{|c|}{ Staggered grid. } \\
\hline $1 / w$ & $2 \mathrm{D}$ & $4 \mathrm{D}$ & 6D \\
\hline $2 \mathrm{D}$ & 1.29 & 0.23 & 1.11 \\
\hline $2 \mathrm{D}$ & 2.15 & 0 & 0.41 \\
\hline $6 \mathrm{D}$ & 2.86 & 0.08 & 0 \\
\hline $20 \mathrm{D}$ & 2.37 & 2.27 & 0.47 \\
\hline
\end{tabular}

\section{Staggered grid}

In general, a staggered grid results in a smaller loss of power compared to an aligned grid (Table 2(b)). Again the wave height increase at the edges of the individual wake results in some cases in positive interactions between the wakes. Consequently, the power loss is not always decreasing with increasing in-between distance.

Lay-outs with $w=4 \mathrm{D}$ and $l=2 \mathrm{D}, 4 \mathrm{D}$ and 6D, respectively, and with $w=6 \mathrm{D}$ and $l=4 \mathrm{D}, 6 \mathrm{D}$ and 20D, respectively, have the highest power absorption. Again, a lateral distance of 4D is preferable to $6 \mathrm{D}$, when calculating the absorbed power per $\mathrm{km}^{2}$ (Table 3(b)). Furthermore, a smaller longitudinal distance results in a higher power absorption per $\mathrm{km}^{2}$. The absorbed power per $\mathrm{km}^{2}$ for $w=4 \mathrm{D}$ and $l=$ 4D increases by $2293 \mathrm{~kW} / \mathrm{km}^{2}$ for irregular long-crested incident waves with $H_{s}=1.5 \mathrm{~m}$ and $T_{p}=6.5 \mathrm{~s}$ and by $6006 \mathrm{~kW} / \mathrm{km}^{2}$ for irregular long-crested waves with $H_{s}=2 \mathrm{~m}$ and $T_{p}=7.8 \mathrm{~s}$. For these longcrested waves, a variation of the capture ratio proportional to the variation in Fig. 6 for irregular longcrested waves with $H_{s}=1 \mathrm{~m}$ and $T_{p}=5.2 \mathrm{~s}$ has been assumed. Even a higher power absorption per $\mathrm{km}^{2}$ is expected for short-crested waves as the wake behind a WEC in the farm is shorter due to a faster regeneration behind the WEC.

To select the best lay-out the cost per unit of installed power should be considered as well. The latter aspect will be studied in further research (Beels et al., 2010c).

\begin{tabular}{|c|c|c|c|}
\hline (a) & \multicolumn{3}{|c|}{ Aligned grid. } \\
\hline $1 / w$ & $2 \mathrm{D}$ & $4 \mathrm{D}$ & $6 \mathrm{D}$ \\
\hline $2 \mathrm{D}$ & 2357 & 1393 & 955 \\
\hline $2 \mathrm{D}$ & 1622 & 902 & 645 \\
\hline $6 \mathrm{D}$ & 1232 & 699 & 494 \\
\hline $20 \mathrm{D}$ & 361 & 273 & 200 \\
\hline (b) & \multicolumn{3}{|c|}{ Staggered grid. } \\
\hline $1 / w$ & $2 \mathrm{D}$ & $4 \mathrm{D}$ & 6D \\
\hline $2 \mathrm{D}$ & 3037 & 2075 & 1333 \\
\hline $2 \mathrm{D}$ & 1619 & 1279 & 871 \\
\hline $6 \mathrm{D}$ & 1037 & 904 & 656 \\
\hline $20 D$ & 373 & 227 & 206 \\
\hline
\end{tabular}

\section{CONCLUSIONS}

In this paper the wake effects behind a single and multiple hypothetical WECs of the overtopping type have been studied in a time-dependent mild-slope equation model MILDwave.

The dimensions and the magnitude of the wake depend on the WEC specifications and on the incident wave climate. The wake increases with decreasing wave period and decreasing directional spreading.

The power absorption of two lay-outs with nine hypothetical WECs, an aligned and a staggered grid, with increasing lateral (2D, 4D and 6D with $\mathrm{D}=$ device width) and longitudinal $(2 \mathrm{D}, 4 \mathrm{D}, 6 \mathrm{D}$ and 
20D) distances, has been calculated for irregular long-crested waves with $H_{s}=1 \mathrm{~m}$ and $T_{p}=5.2 \mathrm{~s}$. The capture ratio of each WEC in the farm has been adapted to its surrounding wave climate. In general, a staggered grid results in a higher power absorption. A lateral distance of 4D is preferred in comparison to $2 \mathrm{D}$, as the amount of absorbed power is higher, and in comparison to $6 \mathrm{D}$, as the amount of absorbed power per $\mathrm{km}^{2}$ is higher. A smaller longitudinal distance results in a higher amount of absorbed power per $\mathrm{km}^{2}$ as well. The final in-between distances should result in an optimum between the amount of absorbed power per $\mathrm{km}^{2}$ and cost per installed $\mathrm{kW}$.

\section{ACKNOWLEDGMENTS}

Research funded by Ph.D. grant of the Institute for the Promotion of Innovation through Science and Technology in Flanders (IWT-Vlaanderen), Belgium.

\section{REFERENCES}

Beels, C., P. Troch, G. De Backer, M. Vantorre, and J. De Rouck. 2010. Numerical implementation and sensitivity analysis of a wave energy converter in a time-dependent mild-slope equation model, Coastal Engineering, Vol. 57(5), pp. 471-492.

Beels, C., P. Troch, K. De Visch, J.P. Kofoed, and G. De Backer. 2010b. Application of the timedependent mild-slope equations for the simulation of wake effects in the lee of a farm of Wave Dragon wave energy converters. Renewable Energy, 35 (2010) 1644-1661.

Beels C., P. Troch, J.P. Kofoed, P. Frigaard, J. Vindahl Kringelum, P. Carsten Kromann, M. Heyman Donovan, J. De Rouck and G. De Backer. 2010c. A methodology for production and cost assessment of a farm of wave energy converters. Accepted for publication in Renewable Energy.

Booij, N. 1983. A note on the accuracy of the mild-slope equation, Coastal Engineering, 7, 191-203.

Booij, N., I. Haagsma, L. Holthuijsen, A. Kieftenburg, R. Ris, A. van der Westhuysen, and M. Zijlema. 2004. Swan cycle iii version 40.41 user manual (online), accessed 7 April 2005 ed. TU Delft. Available from http://fluidmechanics.tudelft.nl/swan/default.htm.

Brorsen, M., and J. Helm-Petersen. 1998. On the reflection of short-crested waves in numerical models, Proceedings of the 26th International Conference on Coastal Engineering, 394-407.

Cruz, J. 2008. Ocean Wave Energy, current status and perspectives. Springer.

Goda, Y., and Y. Suzuki. 1975. Computation of refraction and diffraction of sea waves with Mitsuyasu's directional spectrum, Tech. Note of Port and Harbour Res. Inst., 230.

Larsen, J., and H. Dancy. 1983. Open boundaries in short wave simulations - a new approach, Coastal Engineering, 7, 285-297.

Lee, C., and K. Suh. 1998. Internal generation of waves for time-dependent mild-slope equations, Coastal Engineering, 34, 35-57.

Lee, C., and S. Yoon. 2007. Internal generation of waves on an arc in a rectangular grid system, Coastal Engineering, 54, 357-368.

Liu, Z., and P. Frigaard. 1997. Random Seas Tech. rep., Hydraulics and Coastal Engineering Laboratory, Aalborg University.

Mansard, E. P. D., and E. R. Funke. 1980. The measurement of incident and reflected spectra using a least squares method, Proceedings of the 17th International Conference on Coastal Engineering, 1, 154-172.

Millar, D., H. Smith, and D. Reeve. 2006. Modelling analysis of the sensitivity of shoreline change to a wave farm, Ocean Engineering, 34, 884-901.

Mitsuyasu, H., F. Tasai, T. Suhara, S. Mizuno, M. Ohkusu, T. Honda, and K. Rikiishi. 1975. Observations of the Directional Spectrum of Ocean Waves Using a Cloverleaf Buoy, Journal of Physical Oceanography, 5, 750-760.

Radder, A., and M. Dingemans. 1985. Canonical equations for almost periodic, weakly nonlinear gravity waves, Wave Motion, 7, 473-485.

Suh, K. D., C. Lee, Y.-H. Park, and T. H. Lee. 2001. Experimental verification of horizontal twodimensional modified mild-slope equation model, Coastal Engineering, 44, 1-12.

Troch, P.. 1998. A numerical model for propagation and transformation of linear water waves, Department of Civil Engineering, Ghent University.

Venugopal, V., and G. Smith. 2007. Wave climate investigation for an array of wave power devices, Proceedings of the 7th European Wave and Tidal Energy Conference, IST. ISBN 978-989-950793-7. 